\title{
Quantitative phase determination by using a Michelson interferometer
}

\author{
Juan A Pomarico ${ }^{1}$, Pablo F Molina ${ }^{2}$ \\ and Cristian D' Angelo \\ IFAS-Universidad Nacional del Centro de la Pcia. de Buenos Aires, Pinto 399, \\ B7000GHG Tandil, Buenos Aires, Argentina \\ E-mail: juanp@exa.unicen.edu.ar
}

Received 25 April 2007, in final form 21 May 2007

Published 6 July 2007

Online at stacks.iop.org/EJP/28/797

\begin{abstract}
The Michelson interferometer is one of the best established tools for quantitative interferometric measurements. It has been, and is still successfully used, not only for scientific purposes, but it is also introduced in undergraduate courses for qualitative demonstrations as well as for quantitative determination of several properties such as refractive index, wavelength, optical thickness, etc. Generally speaking, most of the measurements are carried out by determining phase distortions through the changes in the location and/or shape of the interference fringes. However, the extreme sensitivity of this tool, for which minimum deviations of the conditions of its branches can cause very large modifications in the fringe pattern, makes phase changes difficult to follow and measure. The purpose of this communication is to show that, under certain conditions, the sensitivity of the Michelson interferometer can be 'turned down' allowing the quantitative measurement of phase changes with relative ease. As an example we present how the angle (or, optionally, the refractive index) of a transparent standard optical wedge can be determined. Experimental results are shown and compared with the data provided by the manufacturer showing very good agreement.
\end{abstract}

\section{Introduction}

In describing the wave nature of light [1], interferometry is a basic topic in every optics course. Several configurations have been proposed, such as Young, Michelson, Mach Zender, to mention but a few, which are described in any textbook [2]. The mutually interfering light beams give rise to a spatial modulation of the resulting light intensity, generally known as a fringe pattern. All these instruments have found one of their main applications in the

1 Researcher from CONICET, Argentina.

2 Fellow CONICET, Argentina. 


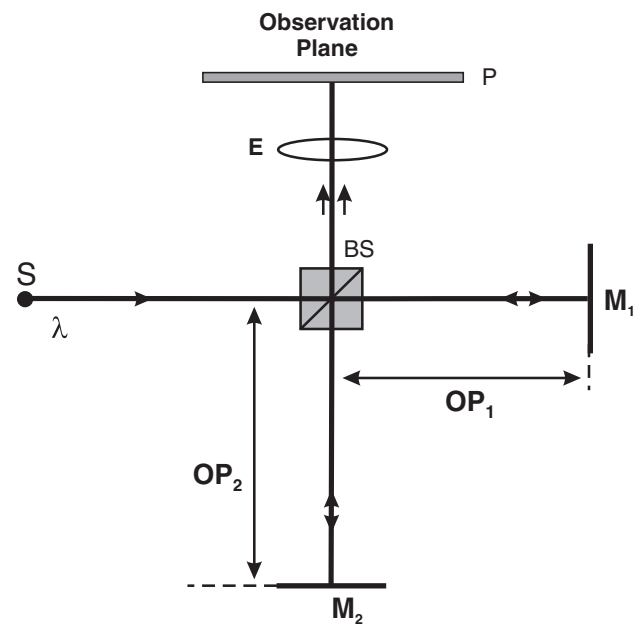

Figure 1. Experimental setup. Light from a coherent source, $S$, is separated in two beams by the beam splitter $B S$. Each beam travels to a mirror $\left(M_{1}\right.$ and $\left.M_{2}\right)$ and is reflected back towards $B S$. Both beams recombine at $B S$ after travelling different optical paths, $O P_{1}$ and $O P_{2}$. The resulting interference pattern is seen at the observation plane after expansion by the $10 \times$ microscope objective, $E$.

determination of the quality of optical surfaces or the measurement of phase objects [3]. Information is usually displayed as a shift or deformation of an initial fringe pattern, taken as a reference, when the object to be evaluated is not present. A very important feature of interferometric arrays is their extreme sensitivity, since scale is fixed by the wavelength of light. That means that any optical path variation which is large compared to the wavelength being used will produce an important change in the reference fringe pattern, a situation which may require sophisticated (electronic) detection techniques.

In this communication we propose a setup for quantitative use of interferometry without using any sophisticated detection electronics. For this purpose it is mandatory to turn down the sensitivity of the interferometer, and it will be shown that a transparent wedge having a small deviation angle used as the phase object to be measured can do this job very well. Modifications of the initial fringe pattern can thus be easily followed by the naked eye, and a quantitative measurement of the wedge angle (or alternatively its refractive index) can be obtained with optical components which are found in every laboratory of undergraduate level physics courses.

In particular, we present our treatment for the Michelson interferometer, for which very interesting applications and teaching strategies can be found in many publications [4-9]. Our approach can also be applied to other types of interferometric devices provided that the calculations are adapted to the particular case.

\section{Theoretical description}

\subsection{Some basic considerations about the Michelson interferometer}

Even though a basic description of the Michelson interferometer can be found in almost every textbook on physical optics, we will describe it here briefly to stress some particular points of interest for our approach. Comments are related to figure 1. In the basic configuration for the 


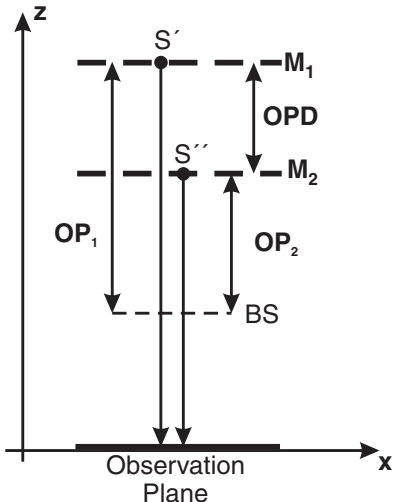

(a)

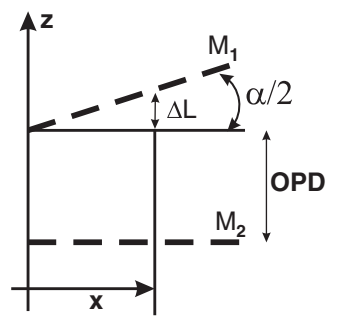

(b)

Figure 2. (a) Optical path difference, $O P D$, for the case of two perfect parallel mirrors. Light propagates along the $Z$-axis and, for this case, the $O P D$ is constant across the mirrors since plane waves are assumed. $S^{\prime}$ and $S^{\prime \prime}$ are virtual sources as seen from the observation plane looking towards $B S$. (b) If one of the mirrors is tilted a small angle with respect to the other $O P D$, and thus, phase change occurs linearly across the mirror. At a given position $x$, an additional optical path $\Delta L$ must be considered. For this sketch it has been assumed that the mirror was tilted only around the $Y$-axis, perpendicular to the picture.

Michelson interferometer, light of wavelength $\lambda$ from a source, $S$, arrives at the beam splitter $B S$ and is divided into two beams, which may be or may be not of equal intensity, depending on the characteristics of the $B S$. Plane wavefronts will be assumed in this description. Beam 1 travels towards mirror $M_{1}$ and is reflected back in the direction of the $B S$. In this round trip it has travelled twice the optical path $\overline{O P_{1}}=n_{1} L_{1}, L_{1}$ being the path length from $B S$ to $M_{1}$ and $n_{1}$ the corresponding refractive index of this branch. Beam 2 makes its way to mirror $M_{2}$ and back travelling along $\overline{O P_{2}}=n_{2} L_{2}$. Both beams recombine at $B S$ and travel now together. The accumulated optical path difference is thus $\overline{O P D}=2\left|\overline{O P_{1}}-\overline{O P_{2}}\right|$ and it gives rise to a phase difference,

$$
\Delta \phi=\frac{2 \pi}{\lambda} \overline{O P D}
$$

between the recombined beams. In equation (1) $\lambda$ is the wavelength in vacuum.

At the observation plane, $P$, the two mirrors appear to lie on the same line of sight, $M_{1}$ at an optical distance $n_{1} L_{1}$ and $M_{2}$ at an optical distance $n_{2} L_{2}$ from $B S$ (see figure 2(a)). The situation can also be described as two virtual sources $S^{\prime}$ and $S^{\prime \prime}$, each one located at the plane of one of the mirrors. If the two mirrors are exactly parallel, as shown in figure 2(a), the phase difference calculated in equation (1) will be constant across the illuminated area of the mirrors and a region of constant brightness is to be expected at the observation plane. However, for the situation shown in figure 2(b), if one of the mirrors is tilted with respect to the other, for example around the vertical axis $Y$, there is an additional and varying phase change along the $X$ direction, perpendicular to the light beam propagating towards the mirror.

Consider, for example, that mirror $M_{1}$ is tilted a small angle $\alpha / 2$ around the $Y$-axis, while $M_{2}$ stays perpendicular to the beam propagation direction. The additional optical path travelled by the beam towards $M_{1}$ and back can be written as

$$
2 \Delta L(x)=2 n_{1} x \operatorname{tg}\left(\frac{\alpha}{2}\right) \simeq n_{1} x \alpha,
$$

$x$ being the distance to the tilt axis. 


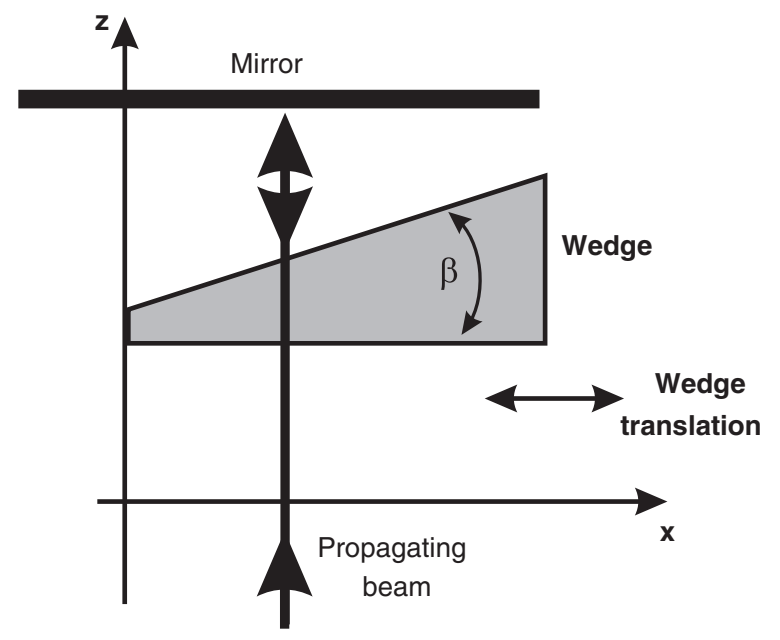

Figure 3. A transparent wedge of index of refraction $n_{\mathrm{w}}$ and with an angle $\beta$ between its faces is introduced in one of the branches of the interferometer. The additional phase change modifies the fringe pattern.

The phase increases now linearly with distance and this situation gives rise to parallel and equally spaced fringes at the observation plane, having a spatial frequency given by $f_{\mathrm{s}}=\alpha / \lambda$. The fringes will present a high contrast if the difference between the optical path lengths travelled by both beams is very small compared to the coherent length of the source [8].

Note also that the occurrence of fringes can be due to the fact that only one of the mirrors is tilted with respect to the direction of beam propagation or that both of them are tilted at different angles, a situation that is, in general, hard to determine.

\subsection{The effect of an additional phase change}

In the following we will suppose that the interferometer is mounted in air $\left(n_{1}=n_{2}=1\right)$ and aligned in such a way that a stable pattern of parallel fringes can be observed, and we will try to quantify some phase changes in one of the branches due to the addition of a phase object in it. The task of measuring phase changes reduces to the one of determining how many fringe periods are shifted with respect to the reference situation for a given position of a phase object (which we want to measure) introduced in one of the branches. Each time $2 \pi$ phase change for the round-trip of the light is completed or, in other words, the optical path has been increased or decremented by $\lambda$, the fringes observed are displaced a whole spatial period. Accordingly with the test object it is possible to observe either local deformations of the pattern or a change of it as a whole. Additionally, for the case of the Michelson interferometer, light travels twice through the phase object, making sensitivity even higher. It is clear that when working with visible light, any optical path variation which is not well controlled will make the fringes move very rapidly, making it impossible to count how many periods were displaced unless some relatively sophisticated electronics are used.

As mentioned in the introduction, we are going to measure the angle of a transparent wedge. This wedge is placed in one of the branches of the interferometer and attached to a linear translation stage, as shown in figure 3 . When it is moved perpendicular to the beam 
propagation direction, a variation of the optical path is introduced for the beam in this branch. The optical path will be increased if the wedge is deeply introduced, and decreased if it is moved away. If the wedge angle is small enough, relatively large lateral displacements (parallel to $X$ direction in figure 3 ) are required to increment the optical path by $\lambda$ and, thus, to see a complete fringe period move in the observation plane.

Consider a wedge of refractive index $n_{\mathrm{w}}$ and angle $\beta \ll 1$ between its faces. If it is displaced a distance $x_{0}$, for example in the direction of the increasing thickness of the wedge, an air thickness

$$
d=x_{0} \operatorname{tg} \beta \simeq x_{0} \beta
$$

will be replaced by the same thickness of glass and the corresponding optical path variation is given by

$$
\Delta \overline{O P_{\mathrm{w}}}(x)=d n_{\mathrm{w}}-d=\beta x_{0}\left(n_{\mathrm{w}}-1\right) .
$$

Each time this path variation equals $\lambda / 2$ a complete fringe period will 'walk' in the observation plane. The 'demagnifying' effect of the wedge is clear from this last equation since $\beta \ll 1$ and thus relative large displacements, $x_{0}$, are required to achieve the condition

$$
\Delta \overline{O P_{\mathrm{w}}}(x)=\frac{\lambda}{2} \equiv \text { one complete fringe. }
$$

Now, if the displacement $x_{0}$ makes $N$ complete fringes to be counted in the observation plane, it results

$$
\beta x_{0}\left(n_{\mathrm{w}}-1\right)=N \frac{\lambda}{2}
$$

then, the angle $\beta$ between the wedge faces can be determined from:

$$
\beta=N \frac{\lambda}{2 x_{0}\left(n_{\mathrm{w}}-1\right)} .
$$

\section{Experimental details}

\subsection{Mounting the interferometer}

As mentioned, quantitative interferometry relies on detecting intensity fluctuations of a given pattern (counting fringe periods in our case) while some phase change is introduced in the interferometer. Because of this it is important to avoid random variations which will cause fringes to move around. To this end, the following general points must be taken into account.

(a) Build the interferometer on a table which is as stable as possible accordingly with your laboratory equipment. An optical table with vibration isolation is not strictly necessary.

(b) Be sure that all components-that is, laser, $B S$, mirrors, etc-are secured in place. Massive or magnetic bases are, in general, a good solution.

(c) It is desirable to keep the length of the branches as small as possible. Large branches tend to magnify distortions and thus cause the fringes to move back and forth. As a practical consideration, branches up to $20 \mathrm{~cm}$ to $30 \mathrm{~cm}$ can be considered as small. This will result in stable patterns while still leaving enough space between $B S$ and the mirrors to introduce, without major trouble, the phase object to be measured. Moreover, almost equal lengths for the branches are preferred to achieve high contrast fringes. 


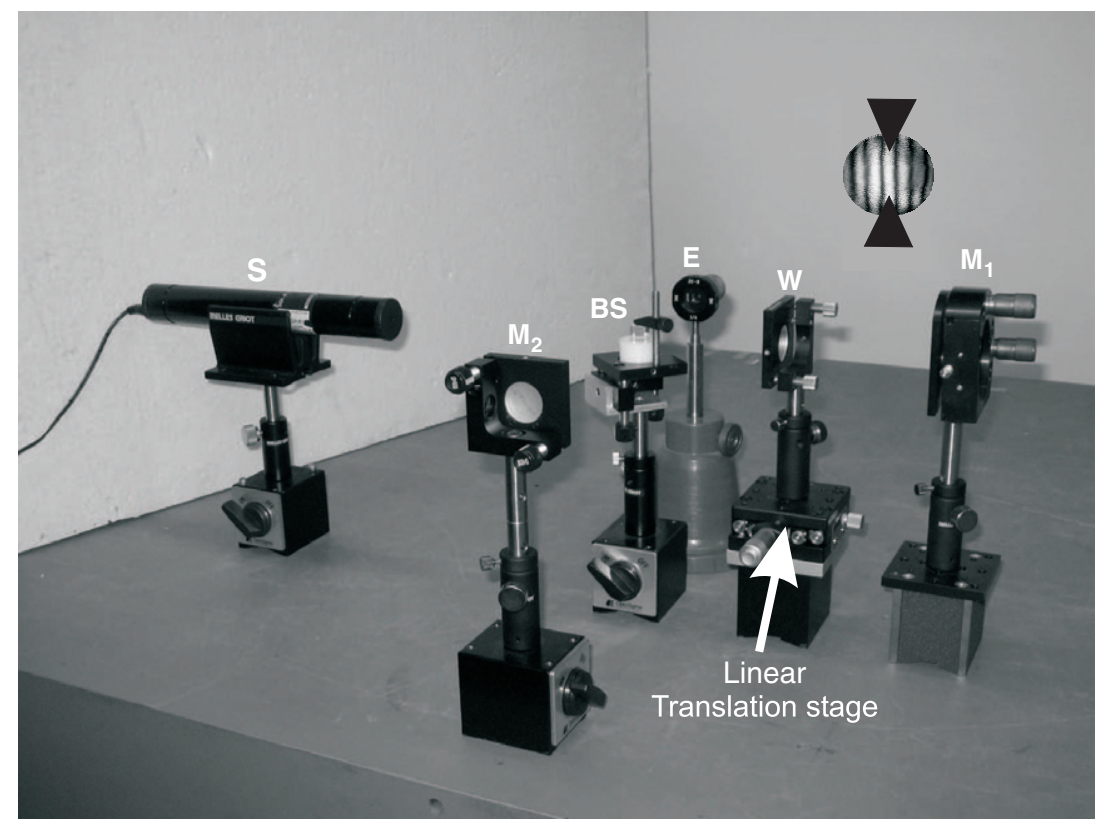

Figure 4. Photograph of the actual experimental setup. A typical fringe pattern was superimposed via photo-montage to illustrate the complete situation observed during the experiment. Optical components are identified as in figure 1 and the wedge prism is labelled $W$.

In our setup we used a $0.5 \mathrm{~mW}$ HeNe laser and the wedge to be tested was a commercial one made of $B K 7\left(n_{\mathrm{w}}=1.51509\right)$ with a deviation angle of $1^{\circ}$, which results in an angle between faces of $\beta=1^{\circ} 56^{\prime}$. The wedge was mounted on a manually actuated linear translation stage with a resolution of $10 \mu \mathrm{m}$. After beam recombination, a $10 \times$ microscope objective was used to expand the fringe pattern and to project it onto a screen placed about $1.5 \mathrm{~m}$ away from the $B S$. One of the mirrors was slightly tilted to achieve a pattern of about five vertical fringes and a couple of dark triangles attached to the screen with their vertex pointing to each other along a vertical line, were used to help the eye to count the fringes as the wedge is displaced.

Care must also be taken in mounting the wedge, since it is to be placed in such a way that the chosen direction of translation produces the maximum increment in thickness per unit displacement. Otherwise it is not the actual angle of the wedge that is measured, but only a component of it. This can be done before mounting the wedge in the interferometer by gently rotating the wedge in-plane and observing at a distant plane the reflection of the laser beam produced by the tilted face of the wedge. The maximum lateral deviation of the reflected beam is the desired condition if the wedge is to be moved horizontally (as shown in figure 3); thus, the incident and the reflected beam define a plane parallel to the working table.

The entire resulting setup is shown in the photograph of figure 4, together with a superimposed picture of a set of fringes.

\subsection{Measurements and error analysis}

Note that equation (4) can be rewritten as

$$
N=\beta \frac{2\left(n_{\mathrm{w}}-1\right)}{\lambda} x_{0}=\tau x_{0} .
$$




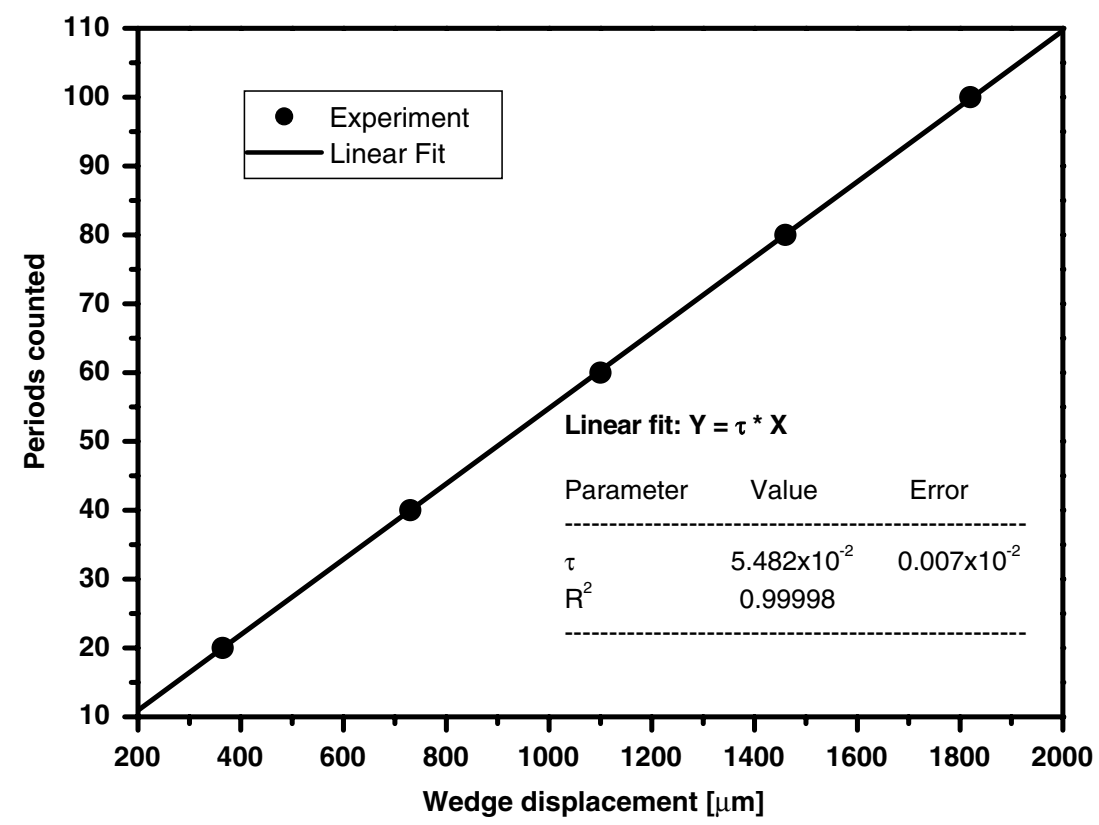

Figure 5. Experimental points (dots) and the corresponding linear fit for them. The resulting regression coefficient of $R^{2}=0.99998$ shows very good agreement between experiment and theory.

The number of fringes $N$ counted for each displacement $x_{0}$ can be plotted as a function of $x_{0}$. The resulting linear plot passes through the origin and has a slope $\tau=\beta \frac{2\left(n_{\mathrm{w}}-1\right)}{\lambda}$, which is proportional to the desired value of $\beta$, which can be easily retrieved provided that the laser wavelength and the refractive index of the wedge are known. Accordingly with this, we have produced several displacements of the wedge prism and counted the corresponding number of complete passing fringes for each situation. Figure 5 summarizes the experimental measurements. The linear fit with a regression coefficient of $R^{2}=0.99998$ shows the quality of the fitting. The slope in figure 5 is given by $\tau=(5.482 \pm 0.007) \times 10^{-2}$, and for $\lambda=632.8 \mathrm{~nm}$ and $n_{\mathrm{w}}=1.51509$, the resulting value for the measured wedge angle is $\beta_{\text {Exp. }}=1^{\circ} 55^{\prime} 46^{\prime \prime} \pm 0.4^{\prime}$ This is to be compared with the value given by the manufacturer, namely $\beta_{\text {Man. }}=1^{\circ} 56^{\prime} \pm 0.5^{\prime}$. Clearly both values, with their respective error bars, overlap.

\section{Conclusions}

In the present work we have demonstrated a practical quantitative application of the Michelson interferometer. The extreme high sensitivity of this device was 'turned down' by using a wedge prism as the object to be measured, providing very small phase changes for a given displacement of the wedge. The setup uses manual translation stages and visual counting of fringes, making it very accessible to undergraduate/elementary optics courses. In spite of this, a high degree of accuracy was achieved in the experiment, resulting in a relative percent error of less than $0.5 \%$, in total agreement with the data provided by the manufacturer. 


\section{Acknowledgments}

The authors would like to thank Professor Dr Héctor F Ranea Sandoval for a critical reading of the manuscript. J Pomarico would like to acknowledge financial support from CONICET Grant PIP 6431.

\section{References}

[1] Born M and Wolf E 1999 Principles of Optics 7th edn (Cambridge: Cambridge University Press)

[2] Hecht E and Zajac A 1986 Optics (Reading, MA: Addison-Wesley)

[3] Malacara D (ed) 1992 Optical Shop Testing 2nd edn (New York: Wiley)

[4] Fritz D J and McLaughlin D J 1993 Optical path difference measurements with Michelson interferometer using a frequency modulated continuos wave ranging technique Am. J. Phys. 61 1028-31

[5] Fox P J, Scholten R E, Walkiewicz M R and Drullinger R E 1999 A reliable, compact, and low-cost Michelson wavemeter for laser wavelength measurement Am. J. Phys. 67 624-30

[6] McKee D K, Nicholls J F H and Ruddock I S 1995 Interferometric measurement of refractive index Eur. J. Phys. 16 127-34

[7] Freschi A A, Caetano N R, Santarine G A and Hessel R 2003 Laser interferometric characterization of a vibrating speaker system Am. J. Phys. 71 1121-6

[8] Verovnik I and Andrej L 2004 The Michelson interferometer-how to detect invisible interference patterns Eur. J. Phys. 25 801-6

[9] Tramannoni F and Matteucci G 2006 Basic experiments of physical optics presented with a modified version of the Michelson interferometer Eur. J. Phys. 27 1267-75 